
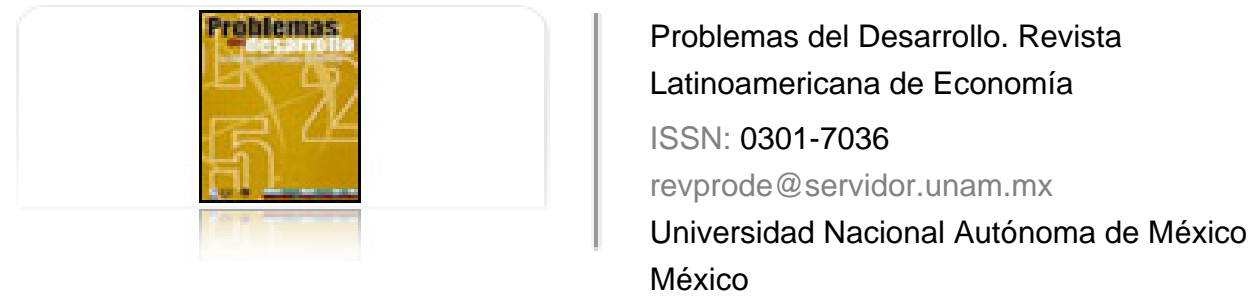

García Garnica, Alejandro; Lara Rivero, Arturo A.

Clúster y coo-petencia (cooperación y competencia) industrial: algunos elementos teóricos por considerar

Problemas del Desarrollo. Revista Latinoamericana de Economía, vol. 35, núm. 139, 2004, pp. 141161

Universidad Nacional Autónoma de México

Distrito Federal, México

Disponible en: http://www.redalyc.org/articulo.oa?id=11825939007

Cómo citar el artículo

- Número completo

- Más información del artículo

Página de la revista en redalyc.org

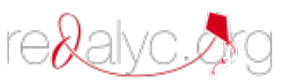

Sistema de Información Científica

Red de Revistas Científicas de América Latina, el Caribe, España y Portugal Proyecto académico sin fines de lucro, desarrollado bajo la iniciativa de acceso abierto 


\title{
CLÚSTER Y COO-PETENCIA (COOPERACIÓN Y COMPETENCIA) INDUSTRIAL: ALGUNOS ELEMENTOS TEÓRICOS POR CONSIDERAR
}

\author{
Alejandro García Garnica* \\ Arturo A. Lara Rivero**
}

Fecha de recepción: 29 de marzo de 2004. Fecha de aceptación: 18 de noviembre de 2004.

\section{Resumen}

Este trabajo tiene como propósito destacar elementos teóricos útiles para analizar distintas formas de colaboración y niveles de competencia entre empresas. Asimismo, se destaca el concepto coo-petencia tecnológica como elemento central en el análisis de clúster o cúmulos industriales.

Palabras clave: clúster, cooperación, competencia, tecnología, red y empresa.

\begin{abstract}
This article aims to highlight the useful theoretical elements for analyzing different types of collaboration and levels of competition between companies. At the same time, it draws attention to the concept technological co-optition as a central element in the analysis of industrial clustering.
\end{abstract}

Key terms: clustering, cooperation, competition, technology, network and company.

Este trabajo forma parte del proyecto colectivo "Agrupamiento y Cooperación Inter firma en el Sector Automotriz. Estudio de los Corredores Toluca-Lerma y Puebla-Tlaxcala", apoyado por el Consejo Nacional de Ciencia y Tecnología (CONACYT). En él participan la UAM Xochimilco, la Universidad Iberoamericana (UI) y la Benemérita Universidad Autónoma de Puebla (BUAP).

**Profesor-investigador en estudios organizacionales, UAM-Xochimilco.Correo electrónico:agg67@hotmail.com

*** Profesor investigador del Departamento de Producción Económica, UAM Xochimilco. Correo electrónico: alara@cueyatl.uam.mx 


\section{Résumé}

Cet article a pour objectif de souligner certains aspects théoriques utiles pour analyser différentes formes de collaboration et de niveau de concurrence entre les entreprises. On met l'accent aussi sur le concept de coo-curence technologique comme un élément central dans l'analyse de clusters ou d'accumulations industrielles.

Mots clés: cluster, coopération, concurrence, technologie, réseau et entreprise.

\section{Resumo}

Este artigo se propõe a destacar elementos teóricos úteis para analisar distintas formas de colaboração e níveis de competência entre empresas. Também, ressaltamos o conceito coopetência tecnológica como elemento central na análise de clusters ou acúmulos industriais.

Palavras-chave: cluster, cooperação, competência, tecnologia, rede e empresa. 


\section{Introducción}

$\mathrm{D}$ esde fines de la década de los setenta y principios de los ochenta, la economía mundial inició la transición hacia una nueva fase de acumulación que ha afectado espacios geográficos, división del trabajo, formas tradicionales de comercialización y producción, así como la cooperaración y competencia entre empresas.

Esta nueva etapa, fase o modalidad histórica del capitalismo se caracteriza por los siguientes elementos: $a$ ) proceso de globalización de las economías que impulsa la generación de encadenamientos productivos en el nivel mundial y el consecuente flujo continuo de diversos productos, insumos y conocimientos en un ambiente de concentración, inestabilidad y asimetría económica, lo cual provoca incertidumbre (Dunning, 1993; Rivera, 1999; y 2000); b) liberación comercial de flujos de capital, inversión extrajera directa, así como privatización — reflejo de cambios institucionales que resultan de la globalización de las economías-; c) impulso de una nueva revolución tecnológica, aparición de nuevos materiales — que acelera la difusión y aplicación de nuevos conocimientos, a partir del desarrollo y convergencia tecnológica entre informática, electrónica y computación, las cuales han facilitado canales de comunicación en los niveles inter e intra empresa (Dunning, 1993; y Ordóñez, 2004)—; y d) fuerte proceso de racionalización económica, el cual se asocia a concentración del capital, inestabilidad financiera, asimetrías regionales, incremento en costos tecnológicos y en la necesidad de elevar la rentabilidad de empresas (Altvater y Mahnkop, 2002).

Mundialización, liberalización, revolución tecnológica y la necesidad de racionalizar la producción son algunas de las fuerzas que están promoviendo cambios en las formas de organización industrial, con las cuales transitan de un esquema de corporaciones multinacionales hacia otro en que se integran nuevos modos de cooperación y competencia empresarial, tanto en el nivel nacional como en el regional (Ernst, 2003).

Este trabajo tiene como propósito fundamental destacar algunos elementos teóricos que contribuyan a enfatizar y analizar la coexistencia de formas de colaboración y competencia tecnológica entre distintas empresas, en el nivel de zonas industriales localizadas. Las preguntas que se intentan responder son: ¿qué son los clúster (cúmulos industriales)?, ¿cuáles son los factores que motivan la competencia capitalista?, y ¿por qué es necesaria la cooperación tecnológica en el nivel inter-empresa?

El trabajo se articula de la siguiente manera: $a$ ) en la primera parte se enfatizan distintos conceptos que aluden a redes industriales o vínculos inter-empresas concentrados en zonas específicas, $b$ ) posteriormente, se plantean definiciones, características y corrientes 
teóricas del proceso de competencia capitalista, $c$ ) después, se describen algunos elementos por considerar en procesos de cooperación tecnológica inter-empresas, así como la importancia que tiene para las compañías promover dichos vínculos, $d$ ) en la cuarta parte, se expone evidencia teórica acerca de procesos de coexistencia de cooperación-competencia en mercados y condiciones específicas, y $e$ ) finalmente, se presentan las conclusiones y la bibliografía.

\section{Distintos espacios geográficos de colaboración empresarial: distritos, conglomerados y clústers}

En últimos años, uno de los fenómenos sociales que más ha llamado la atención de economistas está asociado a la concentración de distritos industriales, ${ }^{1}$ dentro de los cuales surgen redes de colaboración entre diversas organizaciones. Alfred Marshall fue uno de los primeros en analizar las razones por las cuales surge la industria concentrada en ciertas localidades. Marshall (1963) señala algunos elementos que contribuyen a la concentración industrial: 1) condiciones físicas (por ejemplo, naturaleza del clima y suelo), 2) necesidades de consumidores locales (familias y empresas), 3) infraestructura (carreteras), y 4) vías de acceso (transportes). Este autor enfatiza tres aspectos: mutua proximidad de empresas en un distrito industrial, las cuales intercambian información, nuevas ideas e inventos; aparición de actividades o empresas subsidiarias que reducen los costos, y concentración de mano de obra especializada.

Al explicar las causas que contribuyeron al crecimiento y desarrollo de concentraciones o cinturones industriales en Estados Unidos, Paul Krugman coincide con Marshall. Sin embargo, Krugman (1992) agrega que otros aspectos por considerar en la conformación y crecimiento de un cinturón industrial son: rendimientos crecientes a escala, componentes arbitrarios y accidentales, y fenómenos históricos y acumulativos. La convergencia de estos procesos contribuye a la integración de un mercado de mano de obra especializada:

El caso del Cinturón Industrial tiene un interés considerable por sí mismo [...] Más importante que su significado inmediato, no obstante, es lo que la historia de la localización industrial nos enseña sobre la naturaleza de la economía en general. Lo esencial es, por tanto, el hecho de que los rendimientos crecientes a escala y los procesos acumulativos estén muy difundidos y otorguen un papel con frecuencia decisivo a los accidentes históricos [...] es la

\footnotetext{
De acuerdo con Becattini (1988 1989: 7), un distrito industrial es: "un gran complejo productivo, en el que la coordinación entre las distintas fases y el control del funcionamiento regular, no se lleva a cabo mediante reglas preestablecidas y/o mediante mecanismos jerarquizados (como ocurre en la gran empresa privada [...]), sino mediante la confianza en una combinación del juego automático del mercado con un sistema de sanciones sociales impuestas por la comunidad. La proximidad terri torial permite al sistema territorial de las empresas, es decir, al distrito industrial, gozar prácticamente de una economía a gran escala ligada al complejo proceso productivo, sin perder la flexibilidad y la adaptabilidad a las diversas coyunturas del mercado que se desprenden de su fragmentación".
} 
interacción entre rendimientos crecientes e incertidumbre lo que da sentido a la argumentación de Marshall sobre la importancia de un mercado de trabajo conjunto para la localización de las actividades industriales (Krugman, 1992: 30 y 47).

La propuesta de analizar distritos y concentraciones localizadas, también la retomaron Piore y Sabel, quienes aluden a los orígenes de la articulación industrial en el norte de Italia. Los conglomerados industriales surgen por factores asociados a: la cooperación entre pequeñas empresas, en un contexto en el cual ninguna domina permanentemente; la posibilidad de realizar acuerdos informales de corto plazo en el nivel inter-empresa; la conformación de instituciones de apoyo (comerciales, sindicales y financieras) en la zona industrial; y el desarrollo de lazos de comunidad e identidad (Piore y Sabel, 1990).

Para Garud y Van de Ven (1989), la emergencia de nuevas zonas industriales representa la acumulación de los fines emprendidos por una comunidad, en la cual se relacionan simbióticamente actores y empresas para invertir en recursos y transformar invenciones en innovaciones tecnológicas. Esta estructura social e industrial se integra y dinamiza con el desarrollo de subsistemas asociados a los siguientes aspectos: a) institucionales (reglas, regulaciones, legitimación, entre otros), b) materiales (obtención de recursos humanos, financieros, científicos, por mencionar algunos) y c) instrumentales (comercialización, distribución, manufactura y otros). Estos tres subsistemas se interrelacionan mediante intercambio de recursos materiales y humanos, información y conocimientos.

Hay otro tipo de interpretaciones encauzadas a explicar la forma como se comportan los distintos asentamientos industriales de un país, tal es el caso del análisis sectorial. Todo el planteamiento acerca de estrategia competitiva gira en torno al concepto sector económico:

La unidad básica de análisis para comprender la competencia es el sector. Un sector (fabricante o de servicios) es un grupo de competidores que fabrican productos o prestan servicios y compiten directamente unos con otros [...] Dos asuntos sirven de base para la elección de una estrategia competitiva. El primero es la estructura del sector en el que se compite. Los sectores difieren notablemente de la naturaleza de la competencia, y no todos los sectores ofrecen las mismas oportunidades para conseguir una rentabilidad sostenida [...] El segundo asunto esencial en la estrategia es el posicionamiento dentro del sector. Algunas posiciones son más rentables que otras, con independencia de lo que pueda ser la rentabilidad media del sector (Porter, 1991: 63-64).

Estudios posteriores insisten en que el análisis sectorial es limitado porque: 1) se concentra en aspectos cuantitativos o estadísticos, por tanto, se descuidan interconexiones cualitativas ligadas a los flujos de conocimiento surgidas entre redes inter-empresariales, 2) se enfocan solamente grupos de empresas productoras de bienes finales similares, no se consideran vínculos que la empresa puede crear con clientes, proveedores e instituciones especializadas, 3) hay dudas respecto de si las empresas que integran un sector pueden establecer lazos de cooperación con sus rivales, y 4) se busca diversidad en las trayectorias industriales existentes, más que las sinergias (Roelandt y Hertog, 1999). Desde esta perspectiva, 
algunos trabajos reconsideran limitaciones presentadas por el análisis sectorial y tiende a desplazar su objeto de investigación hacia los clúster o cúmulos industriales.

Existen distintas definiciones de clúster o cúmulo. Bockholt y Thuriaux (1999), lo caracterizan como una red en la cual coexisten fuertes lazos de interdependencia — dentro de la cadena productiva de valor- en el nivel de empresas, agentes que producen conocimiento, instituciones puente (consultores) y clientes. Para Roelandt y Hertog (1999) un clúster es conjunto de vínculos e interdependencias entre diversos actores que se involucran en la cadena de valor dedicada a producir bienes, servicios y procesos de innovación.

Por su parte, Porter (1998 y 1999) caracteriza clúster como un grupo específico y geográficamente denso de empresas e instituciones conexas, unidas por rangos comunes y complementarios entre sí. Los cúmulos son formas multi-organizadas y concentradas, integradas por empresas que ofrecen productos o servicios; proveedores de materiales, componentes, maquinaria, servicios especializados e infraestructura; e instituciones financieras, públicas y privadas, que facilitan dinero, capacitación, información, investigación y apoyo técnico especializado.

Por un lado, los cúmulos estimulan la productividad y la innovación tecnológica, lo cual favorece la competencia y la comparación constante entre rivales. Por otra parte, en ellos se captan importantes relaciones y complementariedades asociadas a tecnología, conocimientos prácticos, información, marketing y necesidades de clientes. Los integrantes de un cúmulo comparten necesidades y oportunidades y enfrentan limitaciones y obstáculos a la productividad que les son comunes (Porter, 1999).

En conclusión, en el marco de las definiciones anteriores, consideramos que los cúmulos hacen referencia a zonas geográficas e industriales localizadas, en las cuales un conjunto de empresas e instituciones se interconectan y crean redes intensivas y extensivas de cooperación tecnológica, productiva y servicios, sobre la base de una cadena de valor. En este sentido, emplearemos el concepto red como sinónimo de cúmulo.

\section{De la competencia perfecta a la competencia tecnológica}

$\mathrm{Al}$ investigar el concepto competencia, las definiciones encontradas fueron las siguientes:

Concurrencia de una multitud de vendedores en el mercado libre, que para conseguir la venta de sus productos pugnan entre sí proponiendo los precios que les permitan sus costes, y que se alinean con el mercado (Tamames y Gallo, 1996).

y, por otro lado,

Rivalidad entre los vendedores y los compradores del mismo producto (Echaudemaison, 1996).

Desde este punto de vista, el mercado es una arena donde las empresas luchan y se enfrentan en el ámbito comercial, productivo, financiero y en investigación y desarrollo, 
en un marco legal y regulatorio, con diversas armas estratégicas (Thomas, 1995). La competencia se concibe como un juego sucio en el cual, ante las estrategias de la empresa, los rivales reaccionan con aumento de publicidad y actividades de promoción, disminución de precios o imitación de productos (Bennett, 1992).

Sin embargo, el término competencia puede verse en sentido distinto cuando se le asocia al equilibrio del mercado, como lo hace la teoría neoclásica. En su obra titulada Elementos de Economía Politica Pura o Teoría de la Riqueza Social, León Walras afirmó:

La economía política pura es, en esencia, la teoría de la determinación de los precios bajo un hipotético régimen de competencia libre perfecta [...] esto significa un sistema de competencia libre entre los vendedores de servicios que pujan entre sí a la alza y entre los compradores de productos que pujan a la baja [...] Los mercados mejor organizados desde el punto de vista de la competencia son aquellos en que las ventas y compras se hacen mediante subasta, a través de agentes tales como los agentes de cambio [...] que las centralizan, de tal forma que ningún cambio tiene lugar sin que sus condiciones sean anunciadas y conocidas y sin que los vendedores tengan la oportunidad de rebajar sus precios, y los compradores la de aumentarlos (Walras, 1987:126 y 180).

Este esquema económico considera cinco supuestos: a) los productos que se venden son estandarizados, $b$ ) las empresas aceptan precios del mercado, tienen actitud pasiva, c) los factores de producción (tierra, trabajo y capital) son movibles en el largo plazo, d) productores y consumidores tienen información perfecta, y e) la tecnología es un elemento dado. Así, el mercado es competitivo porque los agentes que integran la oferta y la demanda no pueden influir en los precios, dada su pequeña participación individual en producción o consumo (Frank, 1992 y Varían, 1997).

Como puede observarse, el concepto neoclásico de competencia se aleja de cualquier relación con procesos de rivalidad o dominio de mercado entre empresas. Incluso Marshall intentó eliminar dicho término, al señalar que la competencia se asocia con egoísmo, lucha e indiferencia hacia el bienestar social. Para Marshall (1963), la palabra competencia no refleja lo que sucedía en la sociedad industrial de su época y encerraba aspectos morales negativos. Desde su punto de vista, en la industria y el comercio modernos lo que dominaba era la cooperación, la confianza humana, dada la previsión y la libre elección. En ese sentido, la palabra competencia debía ser sustituida por libertad de industria y empresa o libertad económica.

Sin embargo, Garud y Van de Ven (1989) señalan la necesidad de distinguir entre competencia de precios y tecnológica. En el primer caso se trata de un proceso en el cual empresas rivales ofrecen bienes estandarizados a bajo costo, en un mercado saturado. En cambio, en la competencia tecnológica, la dinámica del mercado se rige por cambios incrementales o rupturas productivas que dan origen a la mejora del producto o al surgimiento de nuevos bienes, en nichos de mercado no saturados. Esta distinción dio origen a la elaboración de múltiples críticas a la interpretación del mercado competitivo. Algunos 
de los planteamientos más importantes al respecto son los de Shumpeter, Demsetz, Winter y Dosi, como se describe a continuación.

Los neoclásicos centran su análisis en esquemas estáticos y estacionarios, que evitan el problema de evolución social o desenvolvimiento económico. Así mismo, dichos teóricos suponen funciones de producción constantes, lo que minimiza, por ende, los cambios discontinuos o espontáneos que caracterizan la producción capitalista, el papel de la innovación y la consecuente utilidad que ésta genera para empresas que la dominan. La innovación es una mutación económica interna surgida de distintas combinaciones de materiales y fuerzas empresariales. La competencia, en este sentido, se explica por la capacidad que tiene cada empresa para generar, de manera continua, nuevos productos y servicios que le aseguren ganancia al empresario (Shumpeter, 1978). Es decir, la competencia capitalista no surge en esquemas rígidos cuyos métodos y formas de organización permanecen constantes; se da en un contexto de destrucción creadora, en el cual aparecen nuevos artículos, técnicas productivas, fuentes de abastecimiento y formas de organización (Shumpeter, 1999).

El modelo de equilibrio de mercado tiene como objetivo explicar cuál es el papel de los precios, no la actividad competitiva. Así, la teoría neoclásica no puede explicar procesos de competencia porque: 1) elimina costos de transacción, 2) no considera el papel de instituciones y autoridades, 3 ) las empresas son entes pasivos al aceptar precios del mercado, 4) se supone información perfecta sin costo y, por tanto, se evade la función del conocimiento especializado en la producción, 5) el equilibrio es estable, a pesar de fricciones existentes en el mercado, y 6) no hay lugar para considerar relaciones conscientes de interdependencia en el nivel inter-empresa o procesos de jerarquía. Para romper con este modelo, es necesario reconocer fricciones del mercado, la consecuente rivalidad y el egoísmo empresarial. También es fundamental analizar cómo compiten las empresas por mejorar la calidad del producto, lograr acuerdos contractuales e innovar (Demsetz, 1986).

Asimismo, la economía del equilibrio general supone un mercado integrado por individuos, productores y consumidores, los cuales poseen racionalidad optimizadora y tienen información plena de todos los precios que existen en el mercado. Sin embargo, se observa que los actores productivos no tienen la capacidad de almacenar y conocer toda la información, su racionalidad es limitada (Simon, 1988). Ante los límites neurofisiológicos, los distintos actores que integran una empresa tienden a cooperar y coordinar sus habilidades y saberes. De esta manera, la organización se convierte en depósito de conocimientos productivos (Winter, 1996). Al coordinarse y dividir el trabajo, las empresas generan rutinas de aprendizaje que les permiten innovar o imitar a otras, así como explotar sus economías de escala de manera específica. La manera como cada empresa aprende e innova define su productividad, la cuota de apropiación de renta y su capacidad para competir en el mercado (Dosi, 1991). 
En el sentido no neoclásico, competencia implica tanto procesos socioeconómicos de cambio y ruptura en estructuras de mercado, como nacimiento y destrucción de empresas. Esta reestructuración de los mercados responde a procesos históricos, es decir, a la acumulación de conocimientos y capacidades tecnológicas específicas, pero también a la adquisición de información; estos procesos coadyuvan a la sobrevivencia y adaptación continua de empresas a las fluctuaciones del entorno. A este respecto señala Freeman (1974):

El mecanismo social de innovación consiste en la supervivencia de los más idóneos. La posibilidad de fracaso de las firmas individuales que intentan innovar surge tanto de la incertidumbre técnica inherente a la innovación como de la posibilidad de juicios erróneos acerca del mercado y de la competencia futuros. La noción de un conocimiento "perfecto" de la tecnología o del mercado se encuentra tan alejada de la realidad de la innovación como lo está la idea de equilibrio.

La reducción de costos, el aumento en productividad, la generación de nuevos y mejores productos son algunas de las formas mediante las cuales se desata una lucha económica y tecnológica entre empresas de un determinado espacio regional, por redistribuir a su favor el mercado y los rendimientos económicos.

En el marco de la estrategia de la empresa se conciben cinco fuerzas competitivas centrales, las cuales determinan la rentabilidad, y la intensidad de la competitividad, y son: $a$ ) poder de negociación con compradores, $b$ ) capacidad de modificar relaciones con proveedores, $c$ ) barreras al ingreso, $d$ ) estrategias de sustitución, y $e$ ) rivalidad. Las tácticas de esta última más utilizadas son: competencia de precios, aumento de publicidad, introducción de nuevos productos y mejora de servicios al cliente (Porter, 1980).

\section{Colaboración y clúster}

Estudios realizados en los niveles teórico y práctico demuestran que en los procesos de innovación exitosos es fundamental la cooperación entre distintos actores u organizaciones. En escasas ocasiones las empresas innovan de manera aislada; en general, se establecen redes tecnológicas y dependencias complementarias de conocimiento científico y técnico entre empresas e instituciones (Roelandt y Hertong, 1999). Algunos de los aspectos que definen las posibilidades de competencia económica y competitividad tecnológica de las empresas son: trayectoria técnica que cada organización sigue, desarrollo de sus capacidades tecnológicas y de procesos de aprendizaje que le son inherentes; posibilidades de desarrollar y participar en redes de cooperación con otras empresas o instituciones que se localizan en su entorno.

Un elemento central por considerar en el análisis del clúster es el de redes interempresariales. Una red se define como:

un conjunto estrecho de vínculos explícitos, selectos y con patrones preferenciales que se dan en un conjunto de empresas a partir de ventajas complementarias y relaciones de 
mercado, teniendo como principal meta la reducción estática y dinámica de la incertidumbre (Freeman, 1991: 501).

Las redes organizacionales surgen por varios motivos: a) necesidad de coordinar procesos de especialización productiva y tecnológica de empresas, $b$ ) introducción rápida y generalizada de nuevas tecnologías de información y comunicación, $c$ ) redescubrimiento de la influencia de las externalidades (estáticas y dinámicas) en los distintos integrantes de un grupo, $d$ ) disminución de la tendencia hacia la integración vertical y auge de los procesos de outsourcing, y $e$ ) innovaciones sociales e institucionales que contribuyan a la solución de problemas socio-económicos complejos, que ni el mercado ni la empresa por sí mismos pueden ofrecer respuesta adecuada (Messner, 1999; Cimoli, 2000; y Altvater y Mahnkop, 2002).

Las relaciones o redes de cooperación entre empresas e instituciones que integran un clúster pueden adoptar distintas formas. Castells (2000) cita cinco: 1) de proveedores, los cuales abarcan convenios de subcontratación, manufactura de equipo original y manufactura de diseño original entre proveedores y clientes, 2) de productores, incluye el acuerdo de coproducción, el cual permite a oferentes, que pueden competir entre sí, compartir recursos, capacidades o segmentos de mercado, 3) de clientes, referidas a la colaboración establecida entre empresas dedicadas a fabricación y distribución de bienes destinados a usuarios finales, 4) coaliciones de normalización, integradas por distintas instituciones y organizaciones con el fin de crear normas globales o estándares de producción y comercialización, y 5) de cooperación tecnológica, ${ }^{2}$ creadas con el objetivo de facilitar la adquisición del diseño de productos y tecnologías de producción o de compartir conocimientos de investigación y desarrollo. ${ }^{3}$

2 Cooperación tecnológica se define como: "aquella relación que vincula a una empresa con otra en aspectos que pueden ayudarla a mejorar o ampliar sus conocimientos respecto a la técnica que emplea y a la forma en que organiza sus recursos [...] La empresa establece una relación de cooperación tecnológica inter firma para hacerse de tecnología (conocimiento), para aprovecharla y adecuarla a sus condiciones específicas. El resultado es la mejoría o ampliación de las capacidades tecnológicas" (Taboada, 2004:90).

3 No hay una clasificación única de redes organizacionales. Por ejemplo, Freeman (1991) destaca los siguientes tipos de acuerdos de cooperación en el nivel inter -organizacional: 1) uniones empresariales y cooperación en investigación, 2) proyectos de investigación y desarrollo, 3) acuerdos de intercambio tecnológico, 4) inversión directa motivada por factores tecnológicos, 5) licenciamientos y acuerdos fuentes, 6) subcontrataciones, distribución de la producción y redes de oferentes, 7) asociaciones de investigación, 8) uniones de programas de investigación patrocinadas por el gobierno, 9) bancos de información computarizada y redes de valor agregado para intercambio científico y 10) redes informales (intercambio de información). En cambio, Sebastián (2000) menciona la siguiente tipología: a) redes de información y comunicación sobre la base de infraestructura electrónica mediante la cual se intercambian distintas clases de datos; $b$ ) redes académicas para intercambiar información e investigación; c) redes temáticas, las cuales se asocian a un tema científico, tecnológico o de gestión; y d) redes de investigación o laboratorios sin paredes enfocadas a actividades de inves tigación y desarrollo tecnológico (Sebastián, 2000). 
Las redes y las consecuentes formas de cooperación que entre distintas empresas e instituciones surgen son transcendentales tecnológicamente porque incrementan canales de aprendizaje, elevan economías de escala y de alcance, posibilitan la flexibilidad y comparten riesgos económicos (Bockholt y Thuriaux, 1999).

Las redes también son viables ahí donde se busca eficiencia e información confiable, en situaciones en las cuales el intercambio no es fácil de cuantificar. Por ejemplo, cuando se transmite el know-how, capacidades tecnológicas, sistemas basados en cero defectos (Powell, 1991). Finalmente, las redes son importantes porque: $a$ ) reducen la incertidumbre tecnológica y de mercado, $b$ ) generan ganancias superávitarias cuando la cooperación técnica es exitosa, $c$ ) reducen comportamiento oportunista al promover entre los participantes el desarrollo de lenguaje común, entendimiento, compromisos mutuos y confianza, $d$ ) coadyuvan a disminuir complejidad y naturaleza intersectorial de nuevas tecnologías; $e$ ) permiten expansión de la producción y entrada de nuevos procesos de trabajo, $f$ ) se obtienen información y conocimientos que contribuyen a monitorear el ambiente, reducir el ciclo de vida de productos o facilitar la entrada a nuevos mercados, y $g$ ) crean y establecen un conjunto de estándares, normas, reglas, sistemas y especificaciones tecnológicas que disminuyen costos de administración y de producción (DeBresson y Amesse, 1991; y Ruiz, 1998).

La estandarización de componentes y procesos coadyuva a reducir la variabilidad en diseños, reducir costos, disminuir la cantidad de información intercambiada y coordinar actividades organizacionales y productivas (Lara, 2001). El tamaño del clúster tecnológico define inversamente el costo de crear un estándar. Los costos se reducen al aumentar el número empresas e instituciones, pues aumentan las economías de escala y en el caso de pocos participantes, se elevan. Sin embargo, si hay gran número de participantes puede debilitar mecanismos de control y alentar oportunismo. Empresas de reciente incorporación al clúster pueden aprovechar el esfuerzo colectivo y desestimular la construcción de más estándares (Lara, 2003).

Pero como ya señalamos, promover la cooperación tecnológica entre empresas también presupone crear bases de confianza. ${ }^{4} \mathrm{Si}$ no existe cierto grado de lealtad y fidelidad entre empresas, se oculta información (Taboada, 2003). Cuando no hay confianza, las empresas no comparten sus saberes por temor a que éstos se difundan a la competencia o se utilicen de forma oportunista. ${ }^{5}$ De cualquier modo, las empresas requieren limitar el

4 Para Porras (2003:213): "la confianza es un elemento fundamental para la colaboración organiza cional [...] donde existe confianza, las organizaciones estarán más dispuestas a colaborar con otras organizaciones en una base de reciprocidad. Más aún las organizaciones tenderán a compartir recursos e información más abiertamente con otros participantes y a reducir el comportamiento oportunista a través del entendimiento mutuo y la buena voluntad de las partes".

5 Además de la confianza, la frecuencia de relaciones y su intensidad y el nivel de especificidad de los activos que se intercambian, otros elementos importantes por considerar en las relaciones de cooperación inter -empresa son: a) reducción de la distancia cognitiva o estructura interpretativa 
intercambio de información a sus proveedores para proteger sus ventajas tecnológicas u organizacionales. La cooperación inter-firma, de ninguna manera elimina la competencia entre ellas por el intento de apropiarse del conocimiento tácito, más bien, la cercanía geográfica y tecnológica tiende a intensificarla (Lara, 1997; y 2001).

Lundvall (1988) afirma que cuando los procesos de aprendizaje son interactivos en el nivel proveedor-usuario, rige la honestidad y la confianza, no el oportunismo y la desconfianza. Sobre todo, durante la relación proveedor-usuario es necesario considerar cuatro dimensiones que se interconectan: a) espacio económico, asociada a la manera como las distintas actividades productivas se localizan en el sistema económico, b) espacio organizacional, relacionado con la integración horizontal y vertical, dentro y fuera de la empresa, c) espacio cultural, se refiere a normas y códigos utilizados para transmitir y codificar información, y d) espacio geográfico, medido en términos de distancia o del espacio en el cual se ubican distintas empresas (Lundvall, 1992). Fristsch y Lukas (1999) sugieren, respecto del espacio geográfico, que los patrones de localización empresarial e institucional pueden favorecer o limitar las relaciones de cooperación. En este sentido, afirman que los "sistemas de innovación poseen una pronunciada dimensión regional".

Las relaciones de cooperación en el nivel inter-empresas también generan costos de transacción, los cuales se asocian a la elaboración y seguimiento de contratos (incluyen redacción, negociación, disputas legales y aseguramiento de compromisos). Incertidumbre, relaciones de intercambio con números pequeños, oportunismo y racionalidad limitada son factores ambientales que afectan las relaciones comerciales entre diversos establecimientos industriales (Williamson, 1991). Desde el punto de vista de la teoría de costos de transacción, los factores específicos que diferencian el tipo e intensidad de transacciones económicas, así como las formas de cooperación son: $a$ ) proximidad espacial, $b$ ) especificidad de los activos físicos, $c$ ) activos humanos, y $d$ ) coinversiones para ampliar planta productiva (Williamson, 1989).

No menos importantes han sido las contribuciones de Alchian y Demsetz al análisis de la cooperación. Estos investigadores sugieren que:

La teoría de la organización económica se enfrenta a dos grandes problemas: explicar las condiciones que determinan si los beneficios que proporcionan la especialización y la producción cooperativa pueden alcanzarse mediante una organización de tipo empresarial, que por medio de los mercados, y explicar la estructura de este tipo de organización (Alchian y Demsetz, 1998).

de la realidad que separa al proveedor del cliente, dada la diferencia de experiencias y conocimientos que cada uno posee como resultado de su trayectoria de vida, y b) la manera en que se construyen las relaciones sociales de cooperación en el tiempo y el contexto histórico en que se dan; c) las normas 
Con relación al problema de las condiciones, se señala que en la interacción de los actores en los niveles inter e intraorganizacional se deben considerar: $i$ ) nivel de eficiencia esperado y obtenido por distintos equipos productivos, ii) control y monitoreo del cumplimiento de los acuerdos y objetivos fijados, iii) vigilancia o supervisión del comportamiento de los equipos, $i v$ ) ventajas y desventajas que ofrecen distintas posibilidades de coordinar e intercambiar información especifica tanto dentro, como fuera de la empresa, y v) tipo de estímulos monetarios existentes (Alchian y Demsetz, 1998).

Particularmente, los incentivos económicos y el conjunto de redes e interacciones que surgen en los cúmulos afectan la competencia porque: $a$ ) incrementan la productividad de empresas o sectores que la integran, $b$ ) eleva su capacidad de innovar, y $c$ ) estimulan la creación de nuevas empresas, lo cual apoya la innovación y expande el cúmulo (Porter, 1998 у 1999).

\section{La coopetencia tecnológica}

Un clúster está integrado por redes espaciales en las cuales conviven organizaciones e instituciones que intercambian información, conocimientos y experiencias productivas, comerciales y tecnológicas. En este espacio territorial, las empresas requieren desarrollar sistemas y estrategias de cooperación que les permitan enfrentar la profunda especialización de trabajo, diversidad tecnológica, altos costos de inversión, incertidumbre del mercado, acortamiento de ciclos de vida de productos y tecnologías, diversos tipos de riesgos financieros y la continua necesidad de aprender. Sin embargo, en este campo geográfico, las empresas también libran diversos enfrentamientos y formas de competencia.

Como señala González (2003:131), al referirse al análisis de mejora tecnológica y a la difusión del conocimiento en redes organizacionales:

Se reconoce que la competencia no es el único modelo de comportamiento que rige los patrones que explican el cambio tecnológico. El papel complementario de la cooperación, la coordinación y la competencia resulta crucial al momento de analizar los patrones de cambio determinantes del éxito o fracaso.

En ese mismo sentido, afirma Cimoli (2000:4):

En los estudios sobre redes se reconoce que la competencia no es el único modelo de comportamiento que explican el cambio tecnológico: el papel de la cooperación, la coordinación y la competencia resulta crucial al momento de analizar los patrones de cambio y las determinantes del éxito o fracaso.

Las transacciones formales e informales de información tecnológica, de saber-hacer, de bienes, recursos financieros y humanos integran uno de los núcleos que explican la coexistencia de redes en los clústers donde de forma paralela se observan procesos de coope- 
ración y competencia entre empresas. Empero, dicha relación se ha modificado en algunos sectores industriales, como el de la informática:

Los sistemas aparecen en todas partes en la tecnología de la información, sistemas operativos y aplicaciones de software [...] Por lo general, una sola compañía no puede pretender ofrecer todas las piezas que conforman un sistema de información. En lugar de ello, los diferentes componentes son fabricados por empresas distintas que usan modelos de producción y gestión muy variados. Las reglas tradicionales de la estrategia competitiva se centran en competidores, proveedores y los clientes. En la economía de la información, las empresas venden componentes complementarios [...] son todos igualmente importantes. Cuando vendemos un componente del sistema, no seremos competitivos si no lo hacemos compatible con el resto del sistema [...] Esta dependencia por parte de las tecnologías de la información de los sistemas significa que las empresas tienen que centrarse no sólo en los competidores, sino también en los colaboradores (Shapiro y Varian, 1998:10).

El término coopetition o coo-petencia integra la tensión y dinámica estratégica que impulsa una empresa para combinar, de manera complementaria, procesos de cooperación y competencia (Nalebuff y Brandenburger, 1996; y Shapiro y Varian, 1998). De acuerdo con Altvater y Mahnkop (2002), el proceso que explica por qué coexiste cooperación y competencia dentro y entre empresas es el siguiente: el incremento de innovaciones tecnológicas en las firmas, si bien aumenta productividad y permite enfrentar la competencia, también eleva la composición orgánica del capital y, por tanto, reduce la tasa de ganancia. Esta caída en rentabilidad, paradójicamente, ha contribuido a reforzar lazos de cooperación y fusiones empresariales, más allá de fronteras nacionales.

Competencia y colaboración tecnológica coexisten porque se presentan en diferentes actores y niveles (Garud y Van de Ven, 1989; y Porter, 2001). ${ }^{6}$ En este sentido, mientras

sociales (sanciones, reglas y normas) institucionales en las que se coopera (García, Lara y Taboada, 2004).

6 La coopetencia es parte de un proceso histórico. Chandler Jr. (1987) describe y analiza factores que coadyuvaron al desarrollo de estrategias de coopetencia en servicios ferroviarios norteamericanos, durante 1870 1880. Este autor observó cómo empresarios e inversionistas dedicados al transporte de mercancías y pasajeros tuvieron necesidad de establecer acuerdos de cooperación informales. Estos vínculos surgieron con el objetivo de reducir costos de transporte, compartir riesgos, aumentar capital social, evitar continuos transbordos de una línea a otra, acortar tiempo en viajes, y ante la necesidad de normalizar cargas, materiales y procedimientos operativos. Chandler Jr. afirma que conforme los vínculos inter empresa crecieron con éxito, también aumentó el nivel de competencia. $\mathrm{Al}$ aumentar el transporte de pasajeros y bienes, se ampliaron las líneas de enlace y red ferroviaria, así mismo, mejoró la estructura organizacional y física de este tipo de transportes. Pero, la necesidad de recuperar inversión exigió elevar el flujo del tráfico y aumentar el número de bienes, y pasajeros transportados, situación que obligó a distintas líneas ferroviarias a promover bajas tarifas, y desarro llar políticas agresivas de venta y publicidad. Este enfrentamiento por ganar mercado comenzó a mermar aún más los beneficios, a tal grado que los inversionistas se vieron obligados a establecer normas que permitieran regular la competencia: "Desde el principio, los directivos del ferrocarril habían considerado la cooperación entre empresas como la mejor manera de controlar la competen cia. En cuanto se ponían en funcionamiento, los ferrocarriles seguían lo que se ha denominado, con acierto estrategia territorial. Al establecer alianzas informales con las vías férreas de enlace y con las 
que dentro de los clúster, la competencia es un estímulo productivo e innovador, las ventajas de la aglomeración de empresas son aprovechadas gracias a múltiples formas de colaboración que pueden llevar a cabo las organizaciones económicas (Qualmann, 1999).

La cooperación de ninguna manera elimina la competencia, antes bien, es una pausa en la acción que incrementa rivalidad empresarial:

La cooperación no conduce a reducir la competencia por dos razones: a) cuando un grupo de competidores forma una alianza para competir más eficazmente, otro grupo enseguida forma su propia alianza. Cada acción tiende a poner condiciones más duras, lo cual conduce hacia niveles más intensivos de competencia; $b$ ) segundo lugar, las alianzas traen costos importantes en términos estratégicos y de organización. Los socios actuales con frecuencia son competidores del mañana, en especial los socios con ventajas competitivas más fuertes o que son más dinámicos (D’Aveni, 1996).

Los niveles que estructurarían dicho proceso de coo-petencia son cuatro: a) mercado, $b$ ) jerarquía, $c$ ) relaciones de cooperación tradicionales, y $d$ ) relaciones de cooperación flexibles. La cooperación tradicional se fundamenta en obediencia, obligación, reciprocidad y lealtad, y regularmente se desarrolla en mercados estables. Por su parte, la cooperación flexible está basada en convenios de cooperación e interés mutuo en reducir costos, intercambiar información y disminuir la incertidumbre de mercados (Okada, 2000). La intensidad o debilidad de las fuerzas que encausan a la cooperación y la competencia en un clúster determinado dependerá del tipo de armonización que predomine (Cuadro 1).

Desde el punto de vista de Okada (2000), el análisis realizado por Williamson, basado en la disyuntiva de jerarquía o mercado, no puede explicar las relaciones de largo plazo ni los procesos culturales. Es decir, las empresas tienen un rango de opciones: incrementar el grado de cooperación, introducir un ambiente de competencia entre las partes que cooperan, o combinar ambas estrategias. En este sentido, las relaciones de largo plazo pueden comprender una mezcla de cooperación y competencia.

La cooperación se apoya en convenios de colaboración que tienen su base en el interés mutuo y el aprovechamiento de interacción entre las partes. Los beneficios esperados mediante la interacción son: altas ganancias por conductas de negocio repetitivas, reducción de costos de transacción, flujo de información, desarrollo de confianza y aumento del apoyo mutuo. Estas prácticas de cooperación están acompañadas de prácticas de monitoreo, evaluaciones, sanciones o la terminación definitiva de relaciones; actividades que al ser llevadas a la práctica introducen la competencia (Okada, 2000).

rivales, los directivos esperaban mantener el flujo de tráfico necesario para garantizar un rendimiento rentable de las inversiones efectuadas en sus líneas [...] Mientras el tráfico de larga distancia se expandía, la estrategia territorial llevada a cabo por las alianzas informales funcionó bien. Pero una vez que su volumen comenzó a disminuir y que se incrementaron las presiones competitivas, los directivos y los propietarios de los ferrocarriles consideraron inadecuadas las alianzas. Entonces, trataron de emplear métodos de cooperación más estrechos y formales con vistas a controlar la competencia. Sólo después de que los intentos de cooperación más concertados y complejos fracasaran, 
Cuadro 1

Mecanismos de armonización

para eficientar las medidas de la promoción,

de la cooperacion y de la generación de la competencia

\begin{tabular}{|c|c|c|}
\hline $\begin{array}{c}\text { Mecanismos } \\
\text { de armonización }\end{array}$ & $\begin{array}{c}\text { Efectividad de } \\
\text { armonización- } \\
\text { medidas de promoción } \\
\text { de la cooperación }\end{array}$ & $\begin{array}{c}\text { Efectividad de } \\
\text { armonización- } \\
\text { medidas de generación } \\
\text { de la competencia }\end{array}$ \\
\hline $\begin{array}{c}\text { Jerarquías } \\
\text { (relaciones intra-empresas) }\end{array}$ & Alta + & Baja \\
\hline $\begin{array}{c}\text { Basada en } \\
\text { cooperación } \\
\text { rígida y tradicional } \\
\text { Basada en } \\
\text { cooperación Flexible }\end{array}$ & Media / Media + & Media / Media + \\
\hline Mercado & Baja & Alta + \\
\hline
\end{tabular}

Fuente: Okada (2000:11)

¿Pero, quién o quiénes definen las estrategias competitivas y la intensidad de cooperación que desarrollan las empresas dentro de distintas estructuras de mercado? La intensidad de cooperación entre empresas e instituciones depende de las estrategias competitivas que desarrollan establecimientos industriales denominados primordiales o dominantes en el sector. Este calificativo se da a las empresas capaces de inducir procesos tecnológicos que delinean el comportamiento y dirección de las redes o el clúster al cual éstas pertenecen. En este sentido, el propósito de la empresa dominante es detentar el núcleo tecnológico del clúster, al administrar, incidir y estimular a proveedores de primera y segunda línea a desarrollarse tecnológicamente (Lara, 2001).

Para lograr esta vinculación, la empresa primordial debe impulsar la construcción de un lenguaje común dentro de la red, coordinar de manera continua sus actividades tecnológicas y las de sus proveedores, invertir en tiempo y dinero, así como difundir procesos de aprendizaje (Lara, 2001a). De este modo, se afirma que las empresas líderes o primordiales que dominan el mercado tratarán de imponer: 1) formas de coordinación más adecuadas a su cadena de valor, 2) niveles y frecuencia de intercambios y, con ello, el tipo de aprendizaje, y 3) medios de apropiarse de la mayor parte del valor agregado generado en la relación cooperativa. Poder y jerarquía dominan las relaciones tecnológicas de cooperación en el nivel inter-empresa (Thompson, 1994; y Godínez, 2000). 
Mediante la colaboración, la empresa primordial puede especializarse, dividir y coordinar los insumos materiales, tecnologías, conocimientos y actores sociales que participan en la cadena de valor de un producto:

La organización económica no puede desarrollar todos los bienes y servicios internamente; necesita complementar sus recursos, técnicas y conocimientos mediante el establecimiento de contratos y redes informales de cooperación con otras entidades. En este proceso de colaboración, la empresa crea vínculos con proveedores, clientes, socios comerciales, instituciones educativas y tecnológicas e incluso con sus competidores. La división del trabajo y la cooperación requieren ser coordinadas (García, 2004).

Por conducto de este proceso de procesos de coordinación, es posible que algunas empresas líderes logren aumentar la eficiencia mediante economías de escala, especialización y redistribución de riesgos, pero también cooptando a competidores (Dunnig, 1993), al contratarlos o realizar coaliciones con éstos. ${ }^{7}$ En un ambiente que se caracteriza por acelerada competencia, las relaciones o vínculos inter e intraorganizacionales se convierten en un factor estratégico para las empresas, el cual puede generar grandes sinergias (Altvater y Mahnkop, 2002).

Este factor estratégico está provocando que las empresas traten de mantener actividades y recursos que son críticos en su cadena de valor, mientras que subcontratan procesos o insumos de tipo secundario. Asimismo, en el contexto estratégico, la empresa debe considerar competidores buenos y malos. Para la empresa, un buen competidor es quien no se muestra complaciente, contribuye a crear equilibrio estable y lucrativo en el nivel sectorial, sin declarar la guerra. Los competidores son buenos en tanto permiten aumentar la ventaja competitiva, mejoran la estructura del sector industrial, contribuyen al desarrollo del mercado y bloquean la entrada de otros competidores (Porter, 1989).

Por otro lado, la estrategia obliga a las firmas a especializarse en un conjunto de actividades y servicios productivos que les permitan mantener una ventaja competitiva en

volvieron en gran número a la creación de redes como medio de eliminar la amenaza de una competencia ruinosa" (Chandler Jr., 1987:196).

7 Los grados de cooperación y compromiso interorganizacionales presentan tres niveles: 1) contrata ción, 2) cooptación y 3) coalición. Esto es, "la contratación se refiere aquí a la negociación de un acuerdo para el intercambio de desempeños en el futuro [...] Los acuerdos contractuales entonces pueden basarse en la fe y en la creencia de que la contraparte se desempeñará de tal modo que man tenga una reputación o prestigio [...] o puede basarse en patrones institucionales mediante los cua les puede dependerse de terceros para evaluar el cumplimiento de las obligaciones y avaluar las sanciones por incumplimiento [...] La cooptación ha sido definida [...] como el proceso de absorción de nuevos elementos en el liderazgo o en la estructura de determinación de políticas de una organización como un medio para prevenir amenazas a su estabilidad o existencia. La cooptación aumenta la certidumbre del apoyo futuro de la organización cooptada [...] La coalición se refiere a una combinación o empresa arriesgada conjunta con otra organización [...] la coalición no sólo suministra una base para el intercambio, sino que también requiere un compromiso con la toma 
el largo plazo, al subcontratar insumos y recursos que no son críticos en sus procesos. ${ }^{8}$ En este sentido, la estrategia de especialización y colaboración está configurando una nueva división interindustrial del trabajo entre los clientes y proveedores que integran un clúster (Ordóñez, 2004).

No obstante, es necesario resaltar que la trayectoria de un clúster no está influida exclusivamente por la estrategia que sigan las empresas primordiales que coordinan las cadenas de suministro. La configuración de los clúster es desigual en los niveles regional o local, y está influida por los siguientes elementos: atmósfera industrial (política industrial, infraestructura, entre otros); nivel de competencia entre grandes y pequeñas empresas; difusión y absorción de métodos de producción de tipo flexible (basados en el justo a tiempo y la administración total de calidad); incertidumbre ambiental; complejidad tecnológica de productos; características específicas de la cadena de suministro (empuje de oferta o demanda) y de clientes y proveedores que la integren; intensidad de las interacciones entre distintos integrantes del clúster; y desarrollo de capacidades locales y aprovechamiento de externalidades (Humphrey, 1995).

\section{Conclusiones}

1. El desarrollo e introducción de nuevos productos y procesos tecnológicos, impulsados en una región geográfica localizada, exigen distintos tipos de conocimientos y recursos materiales específicos, así como formas de colaboración financiera que reduzcan el riesgo y la incertidumbre. La naturaleza de estos vínculos se ubica en la propia división social del trabajo en la cual proveedores, clientes, instituciones públicas y privadas — de investigación y desarrollo- establecen nexos de cooperación tecnológica para complementar sus actividades. Por otro lado, las relaciones de colaboración entre distintas organizaciones producen conflictos de intereses en distintos niveles (estratégicos, productivos, comerciales, por mencionar algunos). Estas contradicciones se exacerban, sobre todo en el nivel inter-empresarial cuando lo que se disputa afecta la trayectoria tecnológica, beneficios y costos, nichos de mercado, conocimientos técnicos acumulados y la posibilidad de ser el primero en innovar, entre otros factores. Es decir, la cooperación se torna rivalidad y competencia industrial cuando se juega la propia supervivencia de la empresa. Sin embargo, también se da el caso en el cual, dentro de un mismo clúster, una empresa logra acuerdos tecnológicos con otras firmas e instituciones y, por otro lado, esa misma empresa se enfrenta a sus rivales en calidad,

conjunta de decisiones [...] Es por lo tanto una forma más restrictiva que la cooptación" (Thompson, 1994, 39 40).

8 Algunas de las ventajas que tiene una empresa al utilizar el outsourcing para adquirir recursos no críticos son las siguientes: a) flexibilidad y facilidad para obtener nuevas tecnologías, artículos de moda o sistemas complejos, b) reducción en el ciclo del diseño al contratar los servicios de firmas que poseen conocimientos especializados, $\mathrm{y}$ c) dispersión de riesgos entre varios proveedores (Quinn y Hilmer, 1995). 
servicio y costo. Pese a ello, la competencia no exime la posibilidad de que empresas rivales lleguen a colaborar de manera conjunta en proyectos tecnológicos de frontera, cuyos resultados aún sean inciertos.

2. Reducir costos, satisfacer necesidades específicas de clientes y producir con calidad son algunos de los requisitos indispensables para mantener la ventaja competitiva, situación que requiere un desarrollo tecnológico continuo. Sin embargo, la mejora de productos y procesos y la incursión en nuevas formas de organización exige cooperación entre empresas e instituciones. Finalmente, el conjunto de redes tecnológicas que se propagan en una zona industrial impulsa a la competencia.

3. La trayectoria evolutiva, desde el concepto industria localizada hasta el de clúster, refleja el tránsito de análisis cuantitativos a propuestas cualitativas, y enriquecen la investigación del conjunto de interrelaciones empresariales e institucionales de una zona geográfica. Este proceso implica transitar de esquemas conceptuales —basados en la interrelación de matrices de insumo-producto en el nivel sectorial y en la medición del valor agregado que cada establecimiento genera en la cadena productiva- a modelos de pensamiento más sistémicos. Enfatizar las problemas y necesidades tecnológicas de los principales promotores organizacionales de la innovación - las empresassignifica considerar aspectos cualitativos en el análisis de redes industriales, tales como intercambio de información, procesos de aprendizaje, oportunismo, confianza, cooperación, jerarquía y estrategias competitivas, entre otros.

\section{Bibliografía}

Alchian y Demsetz, "Producción, Coste de Información y Organización Económica”, en Luis Putterman (coordinador), La Naturaleza Económica de la Empresa, Madrid, Alianza Editorial, 1998, pp. 141-170.

Altvater, Elmar y Birgit Mahnkop, Los límites de la globalización: Economía, Ecología y Política de la Globalización, México, Siglo XXI-UnAM, 2002.

Becattini ,Giacomo, "Los Distritos Industriales y el Reciente Desarrollo Italiano", Sociología del Trabajo, Nueva Época núm. 5, invierno, Madrid, Nueva Época, 1988-1989, pp. 3-17.

Bennett, Roger, "Enderezar Entuertos", en Supervivencia de la Pequeña Empresa, México, CECSA, 1992, pp. 15-29.

Bockholt, Patries y Ben Thuriaux, "Public Policies to Facilitate Clústers: Background, Rationale and Policy Practices in International Perspectiveen Boosting Innovation: The Cluster Approach, OECD Proceedings, Francia”, 1999, pp. 381-409.
Castells, Manuel, La era de la información: la Sociedad Red, vol. núm. 1, México, Siglo xxi, 2000.

Chandler, Alfred Jr., "Cooperación y Competencia en los ferrocarriles, de la Década de 1870 a la de 1880", en La Mano Invisible, Ministerio de Trabajo y Seguridad, Zaragoza, Ministerio de Trabajo y Seguridad, 1987, pp. 179-210.

Cimoli, Mario, "Creación de Redes y Sistema de Innovación: México en un contexto Global”, ElMercado de Valores, México, enero, 2000, pp. 3-17.

D’Aveni, Richard, Hipercompetencia, México, Compañía Editorial Continental, 1996.

DeBresson, Chris y Fernand Amesse, "Networks of Innovators: A Review and Introduction to the Issue", Research Policy 20, North-Holland, 1991, pp. 363-379.

Demsetz, Harold, "Prólogo: la Hipótesis de la Competencia", en La Competencia, Madrid, Alianza Editorial, 1986, pp. 11-27.

Dosi, Giovanni, "Una Reconsideración de las Condiciones y los Modelos del Desarrollo", en 
Pensamiento Iberoamericano, núm. 20, Madrid, 1991, pp. 167-191.

Dunning John, The Globalization of Business: the Challenge of the 1990s, Londres y Nueva York, Routledge, 1993.

Echaudemaison, C. D., Diccionario de Economía, Larousse, Barcelona, 1996.

Ernst, Dieter, "Redes Globales de Producción, Difusión de Conocimiento y Formación de Capacidades Locales", Enrique Dussel, Juan J. Palacios y Guillermo Woo (coordinadores), La Industria Electrónica en México: Problemática, Perspectivas y Propuestas", Guadalajara, Universidad de Guadalajara, 2003, pp. 13-58.

Frank Robert, Microeconomía y Conducta, Mc Graw Hill, Madrid, 1992.

Freeman Cristopher, La Economía de la Innovación Industrial, Madrid, Alianza Editorial, 1974.

_ Research Issue", Research Policy 20, NorthHolland, 1991, pp. 499-514.

Fritsch Michael y Lukas Rolf, "Innovation, Cooperation, and The Region", en Innovation, Industry Evolution and Employment, en David Audretsch y Roy Thurik (editores), Oxford, Cambridge University Press, 1999, pp. 157-181.

García, Alejandro, "La Importancia del Conocimiento y el Aprendizaje en la Ventaja Tecnológica de la Empresa", en Luis Montaño (coordinador), Los Estudios Organizacionales en México, México, UAM-Miguel A, Porrúa-Universidad de Occidente, 2004, pp. 259-280.

García, Alejandro, Arturo Lara y Eunice Taboada, "La Coordinación Híbrida Desde la Perspectivas de Williamson y Nooteboom”, Análisis Económico, vol. XIX, núm. 40, México, UAM-A, primer cuatrimestre, 2004, pp. 101-118.

Garud, Raghu y Andrew Van de Ven, “A Framework for Understanding the Emergence of New Industries", en Research on Technological Innovation, Management and Policy, vol. 4, Nueva york 1989, pp. 195-225.

Godínez, Juan, "Redes Empresariales: Asimetrías y Opciones de Aprendizaje”, en Revista de Análisis Económico, México, UAM-A, primer semestre, vol. Xv, 2000, pp. 165-181.

González, Claudia Rocío, "Redes Organizacionales y Conocimiento: Una Aproximación Conceptual", Gestión y Estrategia, núm. 24, México, UAM-A, julio-diciembre, 2003, pp. 122-134.

Humphrey, John, "Industrial Reorganization in Developing Countries: from Models to Trajectories", Word Development, vol. 23, núm. 1, Oxford, 1995, pp. 149-162.

Krugman, Paul, Geografía y Comercio, Barcelona, Antoni Bosch Editor, 1992.

Lara, Arturo et al., "Intercambio de Información Tecnológica entre Industrias de Automotores y
Autopartes", en Comercio Exterior, vol. 47, núm. 2, México, febrero, 1997, pp. 111-123.

_, "Arquitectura Modular y Evolución de Sistemas Complejos: en el Caso del Sector Automotriz", en José Flores y Ramón Tirado (coordinadores), Economía Industrial y Agrícola en México ante la Apertura, México, UAM-X, 2001a, pp. 201-222.

, "Proveedor exclusivo, Aprendizaje Tecnológico y Clústers", mimeo, Departamento de Producción Económica, México, UAM-X, 2001. , "Redes y Competencia Tecnológica en la Construcción de Estándares", en Revista de Análisis Económico, núm. 38, México, UAM-A, segundo cuatrimestre, 2003, pp. 209-228.

Lundvall, Beng-Ake, "Innovation as an Interactive Process: From User-Producter to the National System of Innovation", en Technical Change and Economic Theory, NuevaYork, Ed. Giovanni Dosi, 1988, pp. 349-369.

_ , "User-Producer Relationships, National Systems of Innovations and Internationalisation", en National Systems of Innovation, Nueva York, Ed. Beng-Ake Lundvall, Pinter Publisher, 1992, pp. 45-67.

Marshall, Alfred, "La Concentración de las Industrias Especializadas en las Localidades Particulares", en Principios de Economía, Madrid, Editorial Aguilar, 1963, pp. 223-232.

Messner, Dirk, "Sociedad de Redes, un Nuevo Modelo de Conducción y Gestión Políticas", Klaus Esser (coordinador), Competencia Global y Libertad de Acción, Caracas, Editorial Nueva Sociedad, 1999, pp. 87-118.

Nalebuff y Brandenburger, Coo-petencia, Grupo Editorial Norma, 1996.

Okada, Yoshita, Competitive-cum-Cooperative Interfirm Relations and Dynamics in the Japanese Semiconductor Industry, Tokyo, Springer, 2000.

Ordóñez, Sergio, “La Nueva Fase de Desarrollo y el Capitalismo del Conocimiento: Elementos Teóricos", Comercio Exterior, vol. 54, núm. 1, México, enero, 2004, pp. 4-17.

Piore Michel y Charles Sabel, The Second Industrial Divide: Possibilities for Prosperity, Nueva York, Basic Books, Inc. Publisher, 1990.

Porras, Salvador, "Consideraciones en Torno a la Confianza y la Colaboración en las Redes Organizacionales", Gestión y Estrategia, México, UAM-A, 2003, pp. 206-217.

Porter Michael, "Análisis Estructural de los Sectores Industriales”, en Estrategia Competitiva, México, CECSA, 1980, pp. 55-94.

___ "Cúmulos y Competencia", en Ser Competitivos: Nuevas Aportaciones y Conclusiones, Ediciones Deusto, Madrid, 1999, pp. 203-288. 
, "Selección de Competidores", en Ventaja Competitiva, CECSA, México, 1989, pp. 218-243.

, "La Ventaja Competitiva de las Empresas en Sectores Mundiales", en La Ventaja Competitiva de las Naciones, Ed. Vergara, 1991, pp. 63-107.

, "Cluster and the New Economics of Competition", en Harvard Business Review, nov-dic., 1998, pp. 77-90.

Powell Walter, "Neither market nor Hierarchy: Network Forms of Organization", Grahame Thompson et al. (editores), Markets, Hierarches and Networks, California, Sage Publications, 1991, pp. 265-276.

Qualmann, Regine, "Distritos Industriales: la Dimensión Local de la Competitividad Internacional", Klaus Esser (coordinador), Competencia Global y Libertad de Acción, Caracas, Editorial Nueva Sociedad, 1999, pp. 135-147.

Quinn, James y Frederick Hilmer, "Outsourcing Estratégico”, Harvard DEUSTo Review, núm. 67, Nueva York, june-august, 1995, pp. 54-69.

Rivera, Miguel Ángel, "Marco Teórico e Histórico para el Estudio de la Reconversión Industrial y el Aprendizaje Tecnológico en México", Miguel A. Rivera (coordinador) Reconversión Industrial y Aprendizaje Tecnológico en México, México, unAm-Facultad de Economía, 1999, pp. 15-50.

- México en la Economía Global: Tecnología, Espacio e Instituciones, México, UNAMUCLA-Jus, 2000,

Roelandt, Theo y Pim Hertong, "Cluster Analysis and Cluster-Based Policy Making in OECD Countries: an Introduction to the Theme", en Boosting Innovation: The Cluster Approach, OECD Proceedings, París, 1999, pp. 9-23.

Ruiz, Clemente, "Redes Industriales: Organización Fundamental de la Economía Globalizada”, El Mercado de Valores, México, febrero, 1998, pp. 3-15.

Sebastián, Jesús, "Las Redes de Cooperación como Modelo Organizativo y Funcional para la I y D”, Redes, vol. 7, núm. 15, Universidad Nacional de Quilmes, Argentina, 2000, pp. 97-111.

Shapiro, Carl y Hal Varian), Information Rules: a Strategic Guide to the Network Economy,
Boston, Massachusetts, Harvard Business School Press, 1998.

Shumpeter Joseph, Teoría del Desenvolvimiento Económico, México, FCE, 1978.

__ "El Proceso de la Destrucción Creadora", en Capitalismo, Socialismo y Democracia, Barcelona, Ediciones Orbis, Biblioteca de Economía, Tomo I, 1999, pp. 118-124.

Simon, Herbert, El Comportamiento Administrativo, Buenos Aires, Editorial Aguilar, 1988.

Taboada, Eunice, "La Credibilidad y la Confianza frente al Oportunismo en las Relaciones de Cooperación Inter firma", Gestión y Estrategia, México, UAM-A, 2003, pp. 197-205.

, ¿Qué hay Detrás de la Decisión de Cooperar Tecnológicamente?: Propuesta de la Explicación de la Cooperación Tecnológica Interfirma con Elementos Teóricos Complementarios, Tesis para obtener el grado de doctor en ciencias económicas, México, Universidad Autónoma Metropolitana, octubre, 2004.

Tamames, Ramón y Santiago Gallo, Diccionario de Economía y Finanzas, Barcelona, Alianza Editorial, 1996.

Thomas, Dan, El Sentido de los Negocios, México, CECSA, 1995.

Thompson James, Las Organizaciones en Acción, México, Mc Graw Hill, 1994.

Varían, Hal, Microeconomía Intermedia: un enfoque Moderno, Barcelona, Antonio Bosch Editor, 1997.

Walras, León, Elementos de Economía Pura o Teoría de la Riqueza Social, Madrid, Alianza Universidad, 1987.

Williamson, Oliver E., "La Economía de los Costos de Transacción", en Las Instituciones Económicas del Capitalismo, FCE, 1989, pp. 26-52.

- "Hacia una Nueva Economía Institucional" y "La Estructura de las fallas de la Organización", en Mercados y Jerarquías: su Análisis y sus Implicaciones, México, FCE, 1991, pp. 17-59.

Winter Sydney, "Coase, la Competencia y la Corporación", en Oliver Williamson y Sydney Winter, La Naturaleza de la Empresa, México, FCE, 1996, pp. 248-270. 